\title{
Risk and resiliency factors associated with poor sleep quality in elderly populations
}

\begin{abstract}
Sleep plays an important role in maintaining overall health and cognition. Additional benefits including memory consolidation have been reported in the literature. For aging adults, these benefits may be compromised by changes having to do with diverse factors including underlying diseases, age-related atrophy, and failing health. In this short review, we describe past and current research on this topic, highlighting $4 \mathrm{P}$ factors that moderate sleep quality in older adults including: Predisposing factors, perpetuating factors, precipitating factors, and protective factors. Although the literature covered in this review is limited due to its narrow focus on elderly populations, there is promising evidence offering potential solutions for anyone experiencing poor sleep quality in their lives.
\end{abstract}

Keywords: aging, poor sleep quality, health, sleep disorders
Volume 6 Issue 3 - 202I

\author{
Rafael De Jesús, William Fishbein \\ Department of Psychology, Laboratory of Cognitive \\ Neuroscience and Sleep, USA
}

Correspondence: Rafael De Jesús, Laboratory of Cognitive Neuroscience and Sleep, Department of Psychology, The City College of New York, I 60 Convent Avenue, New York, NY I003I, USA

Received: May 01, 202I | Published: May 13, 202 I
Abbreviations: mPFC, medial prefrontal cortex; NREM, non-rapid eye movement; SWA, slow save activity; EEG, electroencephalogram; SDB, sleep-disordered breathing; OSA, obstructive sleep apnea; RLS, restless legs syndrome

\section{Introduction}

According to the National Sleep Foundation's 2020 Sleep in America ${ }^{\circledR}$ poll, Americans report feeling sleepy an average of three days a week, with many saying it impacts their daily activities, mood, mental acuity, and productivity. ${ }^{1}$ Among those reporting the highest frequencies of sleepiness, and an increased frequency of naps, are older Americans - with significantly higher proportion increasing with age: $24 \%$ for $75-84$ year-olds as compared to $10 \%$ of $55-64$ year-olds. ${ }^{2}$ Advanced aging, and accompanying health complications, appear to be a major contributor to the poor sleep quality experienced by older adults. Under normal conditions, the circadian rhythm promotes a regular daily cycle of nighttime sleep and daytime wakefulness and alertness. ${ }^{3}$ Aging transforms sleep architecture in a number of ways causing many sleep-related problems that directly impact health and cognition. For example, polysomnographic studies with older adults show that sleep is characterized by a decline in total sleep time and sleep efficiency, ${ }^{4,5}$ longer sleep onset latency, increased waking after sleep onset, and sleep fragmentation. ${ }^{6,7}$ In a study by Mander and colleagues $^{8}$ it was demonstrated that medial prefrontal cortex (mPFC) grey-matter atrophy exhibited in a small sample of older adults contributed to reduced NREM slow save activity (SWA), resulting in impaired long-term memory. Of clinical significance in the elderly, sleep disturbance is associated with decreased memory, impaired concentration, and impaired functional performance. ${ }^{9}$ Studies have confirmed that electroencephalogram (EEG) data in older adults show a decline in slow waves amplitude ${ }^{10}$ and a reduction in density and amplitude of sleep spindles. ${ }^{11}$ This change may be associated with the age-related cognitive impairments reported by older adults resulting from a weakening or disruption of the memory storage processes underlying sleep-dependent memory consolidation. ${ }^{12}$ Sleep is known to play a role in memory formation of newly learned information [for a review see 13]; ${ }^{13}$ a finding reported in the literature to be tied to possible evolutionary advantages..$^{14}$
Considering the important role that sleep plays in everyday life, it should not be dismissed as something that for older adults, is just a normal part of aging. In this short review, we highlight the impact of 4P factors that moderate the effects of sleep quality in older adults including: Predisposing factors; perpetuating factors; precipitating factors; and protective factors.

Predisposing Factors for Poor Sleep Quality. Predisposing factors are defined as "areas of vulnerability that increase the risk for the presenting problem". ${ }^{15}$ There are many conditions that contribute to poor sleep quality in the elderly population. Among the most common ailments affecting older adult's quality sleep are insomnia, sleep-disordered breathing (SDB)—encompassing obstructive sleep apnea (OSA), hypopnea (shallow breaths), and upper airway resistance syndrome - to name a few. ${ }^{16}$ Other sleep-related disorders in the elderly include Restless Legs Syndrome (RLS), periodic limb movement disorder, REM-behavior disorder, and circadian rhythm disorders, which involve either difficulty falling asleep, waking up during the sleep cycle or waking up too early, and being unable to fall back to sleep. ${ }^{5}$ Important to note is the fact that in the elderly oftentimes these conditions do not occur in isolation. There is increasing research suggesting that medical conditions such as diabetes, nocturia, cardiovascular disease, respiratory disorders and chronic pain contribute to poor sleep quality in the elderly. ${ }^{17}$ Older adults are also impacted by other factors associated with aging, such as disease, medication for pain management, changes in the environment, or other co-occurring age-related problems. ${ }^{18}$ The urge to fall asleep, and the sensation of sleepiness, are among the tell-tale signs of the "sleep homeostatic process" or sleep-drive that characterize the compensatory response to sleep loss; an effect that is reflected in the need for older adults to take naps as a way to restore functioning and make up for the sleep-loss or "sleep debt" incurred during night hours. ${ }^{19}$

In fact, as reported by the 2003 Sleep in America poll, among older adults who nap, the frequency of naps increases with age. ${ }^{2}$ As previously mentioned, medical conditions can predispose older adults to poor sleep quality by affecting their physical functioning. Survey responses from 2003 Sleep in America poll, show that about one in 
seven older adults $(15 \%)$ report ambulatory limitations, defined as finding it very difficult or being unable to walk one-half mile without help and/or walk up or down a flight of stairs without help. ${ }^{2}$ These older adults with ambulatory limitations differ from those without these limitations in that they are more likely to: a) sleep less than six hours $(22 \%$ vs. $11 \%)$; b) sleep nine or more hours (9\% vs. $6 \%)$; c) report their sleep quality as fair or poor $(37 \%$ vs. $20 \%)$; d) have a diagnosis of a sleep disorder, such as insomnia, sleep apnea, or restless legs syndrome ( $28 \%$ vs. $11 \%)$; e) report experiencing a symptom of insomnia, such as difficulty falling asleep, waking a lot during the night, waking too early and cannot get back to sleep, and waking up feeling unrefreshed (66\% vs. $44 \%)$; f) report having difficulty falling asleep (33\% vs. $15 \%)$; g) report waking a lot during the night $(50 \%$ vs. $30 \%)$; $)$ report waking too early and cannot get back to sleep ( $38 \%$ vs. $20 \%$ ); i) report waking up feeling unrefreshed ( $43 \%$ vs. $23 \%)$; j) report daytime sleepiness ( $28 \%$ vs. $12 \%)$; k) report unpleasant feelings in their legs ( $39 \%$ vs. $13 \%)$; and l) report having a sleep problem ( $84 \%$ vs. $64 \%){ }^{2}$

Perpetuating Factors for Poor Sleep Quality. Perpetuating factors are defined as "any conditions in the patient, family, community, or larger systems that exacerbate rather than solve the problem". ${ }^{15}$ Counterproductive lifestyle choices and maladaptive habits can contribute to poor sleep quality. The elderly in particular face a number of challenges related to advanced aging. For instance, as reported by Monjan, ${ }^{16}$ "abdominal obesity, elevated triglycerides, low level of high-density lipoprotein cholesterol, high blood pressure and high serum glucose" all can perpetuate or exacerbate poor sleep quality. Monjan ${ }^{16}$ cites a study by Knutson et al..$^{20}$ that underscores the vicious cycle perpetuating poor sleep quality in older adults stating:

A number of studies have shown that sleep loss can lead to impairments in glucose metabolism and insulin resistance as possible precursors to Type 2 diabetes and to alterations in the hormones leptin and ghrelin which then lead to inappropriate appetite increases leading to increased food consumption (p. 3).

These effects along with the following poor sleep hygiene routines also contribute to the continuation of poor sleep quality including: caffeinated beverages (e.g., coffee), alcohol, naps, exposure to electronic devises such as televisions, computers, and cellular phones. ${ }^{21}$ Perpetuating factors for insomnia, a common concern in older adults that contributes to poor quality sleep, as noted Perlis, Shaw, Cano, and Espie ${ }^{22}$ include: "the practice of non-sleep activities in the bedroom, the tendency to stay in bed while awake, and the tendency to spend excessive amounts of time in bed" (p. 852).

Precipitating Factors for Poor Sleep Quality. Precipitating factors are defined as "stressors or other events (they could be positive or negative) that may be precipitants of the symptoms". ${ }^{15}$ Environmental factors and life events contributing to poor sleep quality in the elderly amount to marked daytime dysfunction and irregular sleep-wake times. For instance, as noted by Monjan, ${ }^{16}$ "in the elderly, transient insomnia may be caused by bereavement, adjustment to medical difficulties and physical limitations" (p. 1). Although "age itself does not predict insomnia", ${ }^{23}$ the presence of insomnia and other related sleep disorders remains high in this population due to a variety of common factors encountered later in life. ${ }^{23}$

Costa $^{24}$ reviewed epidemiological and clinical studies documenting that poor sleep quality is compounded by shift work and other stressors in elderly populations. The working population, as he reports, "is engaged in irregular or 'non-standard' working hours, including shift and night work, week-end work, split shifts, on-call work, compressed weeks, telework, part-time work, variable/ flexible working time, and prolonged duty periods (i.e., 12-h shifts) (p. 112). Aging when combined with shift work notes Costa, ${ }^{24}$ "can be underestimated in elderly shift workers due to the 'sick shift worker effect,' that is workers no longer working on shifts due to intervening health disorders that are more likely to occur with ageing" (p. 116). Concerning shift workers $\operatorname{Costa}^{24}$ further reports:

Shift workers often complain about irritability, nervousness, and anxiety in relation to more stressful working conditions and higher difficulties in family and social life. Persistent disruption of circadian rhythms and sleep deficits may lead to chronic fatigue, mood disorders, neuroticism, as well as to chronic anxiety and/or depression, creating a situation where workers have higher absenteeism and often require the administration of psychotropic drugs (sedatives and hypnotics) (p. 115).

Another significant precipitant to poor sleep quality is being overweight; as reported by Monjan, ${ }^{16}$ "the most important risk factor for SDB is overweight and obesity, suggesting that its incidence is likely to increase dramatically with the recent epidemic of overweight and obesity" (p. 2). The development of a "night owl" pattern, although less common in older adults, contributes to bedtimes delayed until the early morning hours. ${ }^{25}$ These alterations in sleep-wake cycles as Neubauer ${ }^{25}$ reports, "may have been tolerated, in the younger years during employment, when the cues of early morning bright light were stronger...On retirement, however, these cues weaken, and the sleepwake cycle may become delayed by several hours" (p. 2552).

Protective Factors for Poor Sleep Quality. Protective factors are defined as "the patient's own areas of competency, skill, talents, interest and supportive elements". ${ }^{15}$ Proactive and adaptive contributors that safeguard against poor sleep quality in elderly populations often include incorporating effective sleep hygiene techniques into their lifestyle. As reports Neubauer ${ }^{25}$ "implementation of good sleep habits and daily physical activity should help create an environment conducive to restorative sleep" (p. 2555). In a study with 74 volunteers $(52.7 \%$ women; aged $53.7 \pm 5.1)$, higher levels of overall physical activity, and muscular strength were related to better subjective sleep quantity and quality. ${ }^{26}$ Weight loss has been reported to reduce the progression of SDB, contributing to improved sleep quality. ${ }^{27}$ The value of daily exercise and exposure to daylight as reports Neubauer ${ }^{25}$ "can help reinforce the circadian cycle" (p. 2555). In addition, temporary use of sleep-promoting medications including low-dose sedatives "are helpful in patients with depressive symptoms" (p. 2555). Additional sleep hygiene measures (Table 1) including the use of non-prescription medications such as antihistamines, melatonin, and other herbal medications (e.g., valerian, chamomile, hops, kavakava, and passionflower) have been well-used sleep aids; although caution should be used especially in elderly populations considering there are limited controlled trials demonstrating efficacy and safety. ${ }^{9}$

Table I Sleep-hygiene measures

Avoid and minimize use of caffeine, cigarettes, stimulants,

alcohol, and other medications

If medically able, increase activity level in the afternoon or

early evening (not close to bedtime) by walking or

exercising outdoors

Increase exposure to natural light and bright light during

day and early evening 
Table Continued...

Avoid napping, particularly after 2:00 PM; Limit naps to I nap
of less than 30 minutes
Check the effect of medications on sleep
Go to bed only when sleepy
Maintain comfortable temperature in bedroom
Minimize light and noise exposure as much as possible
Eat a light snack if hungry
Avoid heavy meals at bedtime
Limit liquids in the evening
Keep a regular schedule
Rest and retire at the same time each day
Eat and exercise on a regular schedule
Stress-management measures:
Dolerance of occasional sleeplessness

Source: Kamel NS, Gammack JK. Insomnia in the elderly: Cause, approach, and treatment. The American Journal of Medicine. 2006; I 1 9:463-469.

\section{Limitations and conclusion}

A significant limitation in our review is the fact that we discussed factors that are not exclusive to elderly populations; many of these influencing factors mentioned are also seen in young and middleaged-adults. Therefore, our review in retrospect should have taken a more nuanced or narrow focus to make a stronger case for associating these factors to older adults. Another limitation is in our definition and use of "poor sleep quality," which is discussed in general terms and not properly defined or operationalized. To get a better sense of what in fact is "poor sleep quality" we should have made an effort to: a) distinguish between what is considered optimal sleep quality versus "poor sleep quality," and b) operationalized sleep quality in the context of its contemporary definition (e.g., For example, the National Sleep Foundation ${ }^{1}$ uses a Sleep Health Index ${ }^{\circledR}$ as defined by: measures of sleep duration, sleep quality, and disordered sleep).

Through the lens of a stress-diathesis conceptualization approach, we have highlighted and identified factors that help and hinder poor sleep quality in older adults. This short review also addressed findings in the literature related to sleep and aging. Through a survey of past and current research studies, we set out to advance our understanding of the importance of sleep in everyday life, especially, as it relates to aging populations.

\section{Acknowledgments}

The authors wish to thank the past members of the Laboratory of Cognitive Neuroscience and Sleep.

\section{Conflicts of interest}

The authors declare that there is no conflict of interest.

\section{References}

1. National sleep foundation. sleep in america Poll 2020: Americans feel sleepy 3 days a week, with impacts on activities, mood and acuity. 2020.

2. National sleep foundation. 2003 Sleep in America Poll. 2003.

3. Carskadon, MA, Dement WC. Monitoring and staging human sleep. In: MH Kryger, T Roth, et al. editors. Principles and practice of sleep medicine, 5th edn,. St. Louis: Elsevier Saunders. 2011:16-26.

4. Ohayon MM, Carskadon MA, Guilleminault C, et al. Meta-analysis of quantitative sleep parameters from childhood to old age in healthy individuals: developing normative sleep values across the human lifespan. Sleep. 2004;27(7):1255-1273.

5. Bliwise, DL, Scullin MK. Normal aging. In: MH Kryger, T Roth, editors. Principles and practice of sleep medicine, $6^{\text {th }}$ edition,). Philadelphia: Elsevier. 2017:25-38.

6. Bliwise DL, Foley DJ, Vitiello MV, et al. Nocturia and disturbed sleep in elderly. Sleep Medicine. 2009;10(5):540-548.

7. Pace-Schott EF, Spencer RMC. Age-related changes in the cognitive function of sleep. Progress in Brain Research. 2011;191:75-89.

8. Mander BA, Rao V, Lu B, et al. Prefrontal atrophy, disrupted NREM slow waves and impaired hippocampal-dependent memory in aging. Nature Neuroscience. 2013;16:357-364.

9. Kamel NS, Gammack JK. Insomnia in the elderly: Cause, approach, and treatment. The American Journal of Medicine. 2006;119:463-469.

10. Carrier J, Viens I, Poirier G, et al. Sleep slow wave changes during the middle years of life. European Journal of Neuroscience. 2011;33:758766.

11. De Gennaro L, Ferrara M. Sleep spindles: an overview. Sleep Medicine Reviews. 2003;7:423-440.

12. Harand C, Bertran F, Doidy F, et al. How aging affects sleep-dependent memory consolidation?. Frontiers in Neurology. 2012;3:Article 8.

13. De Jesús R, Fishbein W. The influence of sleep on emotional memory consolidation processes. Archives Of Psychology. 2018;2(5).

14. Fishbein W, Lau H, De Jesús R, et al. Sleep, neural reuse, and memory consolidation processes. Behavioral and Brain Sciences. 2010;33(4):273273.

15. Campbell, WH, Rohrbaugh RM. The biopsychosocial formulation manual: A guide for mental health professionals. CRC Press. 2006.

16. Monjan AA. Perspective on sleep and aging. Frontiers in Neurology. $2010 ; 1: 124$

17. Bliwise, DL, King AC, Harris RB, et al. Prevalence of self-reported poor sleep in a healthy population aged 50-65. Social Science \& Medicine. 1992;34:49-55.

18. Ancoli-Israel S, Cooke J.R. Prevalence and comorbidity of insomnia and effect on functioning in elderly populations. Journal of the American Geriatrics Society. 2005;53:S264-S271.

19. Goldman SE, Hall M, Boudreau R, et al. Association between nighttime sleep and napping in older adults. Sleep. 2008;31(5):733-740.

20. Knutson, KL, Rathouz PJ, Yan LL, et al. Intra-individual daily and yearly variability in actigraphically recorded sleep measures: The CARDIA study. Sleep. 2007;30(6):793-796.

21. Shochat T. Impact of lifestyle and technology developments on sleep. Nature Science Sleep. 2012;4:19-31.

22. Perlis M, Shaw PJ, Cano G, et al. Models of insomnia. In: MH Kryger, T Roth, editors. Principles and Practices of Sleep Medicine, $5^{\text {th }}$ edn, St. Louis, MO: Elsevier. 2011:850-865. 
23. Foley D, Monjan A, Brown SL, et al. Sleep complaints among elderly persons: an epidemiologic study of three communities. Sleep. 1995;18:425-432.

24. Costa, G. Shift work and health: current problems and preventive actions. Safety Health Work. 2010;1:112-123.

25. Neubauer DN. Sleep problems in the elderly. American Family Physician 1999;59(9):2551-2558.
26. Mochón-Benguigui, S, Carneiro-Barrera A, Castillo MJ, et al. Role of physical activity and fitness on sleep in sedentary middle-aged adults: the FIT-AGEING study. Scientific Reports. 2021;11:539.

27. Peppard PE, Young T, Palta M, et al. Prospective study of the association between sleep-disordered breathing and hypertension. New England Journal of Medicine. 2000;342:1378-1384. 